\title{
Parity-time-symmetric coupled asymmetric dimers
}

\author{
L. Jin* \\ School of Physics, Nankai University, Tianjin 300071, China
}

(Received 31 October 2017; published 19 January 2018)

\begin{abstract}
We investigate a parity-time $(\mathcal{P} \mathcal{T})$-symmetric system that consists of two symmetrically coupled asymmetric dimers. The enclosed magnetic flux controls the $\mathcal{P} \mathcal{T}$ phase transition. The system can reenter the exact $\mathcal{P} \mathcal{T}$ symmetric phase from a broken $\mathcal{P} \mathcal{T}$-symmetric phase with large non-Hermiticity. Two-state coalescence may have one or two defective eigenstates. The topology of exceptional points is reflected by the phase rigidity scaling exponents. The topology changes when exceptional points coincide. The geometric phases accumulate when encircling the exceptional points and vary as the magnetic flux. The magnetic flux is favorable for the realization of high-order exceptional points. A triple point of different quantum phases has an order of 4 . The perturbation around the four-state coalescence leads to a fourth-root mode frequency splitting; the sensing sensitivity is significantly enhanced.
\end{abstract}

DOI: 10.1103/PhysRevA.97.012121

\section{INTRODUCTION}

Parity-time $(\mathcal{P} \mathcal{T})$-symmetric non-Hermitian Hamiltonians can possess real spectra; this discovery stimulated a burst of research interest in the extension of quantum mechanics [1-10]. $\mathcal{P} \mathcal{T}$ symmetry is the origin of many intriguing features in non-Hermitian systems. Various optical systems are fruitful platforms for the investigation of $\mathcal{P} \mathcal{T}$ symmetry [11-14]. The phenomena of open quantum systems can be described by $\mathcal{P} \mathcal{T}$-symmetric non-Hermitian Hamiltonians after an average overall decay has been removed $[15,16]$. In a passively coupled waveguide, where losses were asymmetric, $\mathcal{P} \mathcal{T}$-symmetry breaking was demonstrated [17]; the $\mathcal{P} \mathcal{T}$-symmetric coupled waveguide was realized through introducing an active gain; the light intensity oscillated in the exact $\mathcal{P} \mathcal{T}$-symmetric phase and exponentially increased in the broken $\mathcal{P} \mathcal{T}$-symmetric phase [18]. $\mathcal{P} \mathcal{T}$ symmetry was also proposed and experimentally realized in coupled microcavities [19-23]. In a linear region of an active cavity, doped ions under pumping generated a self-adapted net gain that balanced the loss in the corresponding dissipative cavity. The light transport is reciprocal and nonreciprocal in the exact and broken $\mathcal{P} \mathcal{T}$-symmetric phases, respectively [20]. When the gain saturation induces dominating large nonlinearity, the coupled microcavities are nonreciprocal and can be applied as an optical isolator [23].

Recently, research has addressed exceptional points (EPs). The EP in a $\mathcal{P} \mathcal{T}$-symmetric system is the $\mathcal{P} \mathcal{T}$ phase transition point. $\mathcal{P} \mathcal{T}$-symmetric systems with periodical potentials have also been investigated. Unidirectional reflection suppression was demonstrated at the $\mathcal{P} \mathcal{T}$ phase transition point [24]. The

\footnotetext{
*jinliang@nankai.edu.cn

Published by the American Physical Society under the terms of the Creative Commons Attribution 4.0 International license. Further distribution of this work must maintain attribution to the author(s) and the published article's title, journal citation, and DOI.
}

$\mathcal{P T}$ phase transition threshold exists in a $\mathcal{P} \mathcal{T}$-symmetric sinusoidal potential, but any higher degree of non-Hermiticity leads to $\mathcal{P} \mathcal{T}$-symmetry breaking [25]. The competition between two lattice potentials can induce $\mathcal{P} \mathcal{T}$-symmetry breaking and restoration as non-Hermiticity increasing [26]. EPs enhance optical sensing $[27,28]$. A state flip or mode switch occurs when encircling the EPs sufficiently slowly for integer circles. The geometric phases are accumulated in the encircling process and the intriguing topologies of EPs are reflected [29-32]. In a two-level system, when encircling a two-state coalescence exceptional point (EP2), the two eigenenergy levels switch to each other after encircling the EP2 for one circle, and the geometric phase accumulated for each eigenstate is $\pi$ after encircling the EP2 for two circles; to make each eigenstate return to itself, encircling EP2 for four circles is needed [33,34]. High-order EPs have highly complicated topological structures and physical implications [35]; the sensitivity is enhanced due to the cubic-root frequency response near a three-state coalescence exceptional point [36].

Photons, as neutral particles, do not directly interact with magnetic fields. Recent studies have implemented artificial magnetic fields for photons through dynamic modulation of material permittivity, optomechanical coupling, and photonphonon interactions [37-41]. The artificial magnetic field provides a new degree of freedom, which is favorable for optical control and manipulation. In this paper, we study $\mathcal{P} \mathcal{T}$-symmetric coupled asymmetric dimers, where effective magnetic flux is induced by the nonreciprocal coupling of the dimers. We demonstrate that the energy-level structure, the quantum phases, and the topology of EPs are affected by the magnetic flux, the coupling, and the degree of nonHermiticity. We find that the system can reenter an exact $\mathcal{P} \mathcal{T}$-symmetric phase from a broken $\mathcal{P} \mathcal{T}$-symmetric phase at large non-Hermiticity. The gap between two central energy levels vanishes at uniform coupling in the absence of magnetic flux; the chirality of the EPs depends on the competition between the two coupling strengths. An EP2 with two defective eigenstates exists, where a pair of two-state coalescences 
appears. Phase rigidity is a useful measure of state mixing; its scaling exponent characterizes the topology of EPs, which is partly magnetic-flux independent. The topology of EPs changes when they coincide. Four-state coalescence (EP4) appears when an EP2 with one and two defective eigenstates coalesces; the perturbation around the EP4 leads to a fourthroot mode frequency splitting; in that state, sensing sensitivity is substantially enhanced.

\section{II. $\mathcal{P} \mathcal{T}$-SYMMETRIC ASYMMETRIC DIMERS}

We consider two asymmetric dimers symmetrically coupled in a closed configuration; one dimer has gain (green) and the other has loss (red). The system is $\mathcal{P} \mathcal{T}$ symmetric with respect to the left-right reflection, as schematically illustrated in Fig. 1. The parity operation is defined as the space reflection with respect to the $\mathcal{P} \mathcal{T}$-symmetric axis (blue dotted line in Fig. 1).

We investigate the influence of nonreciprocal couplings that induce effective magnetic flux on the spectrum, quantum phases, and topology of EPs. Notably, the magnetic flux as an additional degree of freedom does not break the $\mathcal{P} \mathcal{T}$ symmetry of the system. The magnetic flux provides an alternative way for the control of $\mathcal{P} \mathcal{T}$-symmetric phases and EPs, which may facilitate the application of $\mathcal{P} \mathcal{T}$ metamaterials.

In the coupled resonator system, all the primary resonators possess identical resonant frequency. They are evanescently coupled through auxiliary resonators, the frequency of which is antiresonant with the primary resonators [42]. The optical path-length difference introduced through the coupling process induces a nonreciprocal coupling phase factor, which effectively realizes a synthetic magnetic flux in the closed configuration formed by the four coupled primary resonators. The nonreciprocal extra phase factor in the coupling is $e^{ \pm 2 \pi \Delta x / \lambda}$ for the path-length difference $2 \Delta x$, where $\lambda$ is the resonant wavelength [43]. The interdimer (horizontal) coupling strength

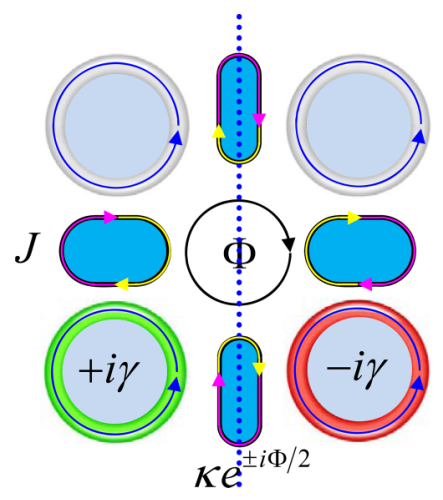

FIG. 1. Schematic of four coupled resonators consisting of two asymmetric dimers. One dimer has loss (red) and the other has gain (green). The coupling strengths are $\kappa$ and $J$. The dotted blue line is the $\mathcal{P} \mathcal{T}$-symmetric axis. The primary (auxiliary) resonators are round (elliptical). The blue arrows indicate the counterclockwise mode supported by the primary resonators. The yellow (magenta) arrows illustrate the path lengths of counterclockwise mode photons traveling in counterclockwise (clockwise) direction between primary resonators. The length difference induces a directional phase factor in the coupling, which results in the nonreciprocity and induces the synthetic magnetic flux $\Phi$. is $\kappa$ with nonreciprocal phase factor $e^{ \pm i \Phi / 2}$; the intradimer (vertical) coupling strength is $J$. The synthetic magnetic flux introduced in the coupled four resonator system equals to $\Phi=4 \pi \Delta x / \lambda$. The gain in the resonator is based on a linear model by assuming a certain gain rate $\gamma$ [22]. The gain in one dimer equals to the loss in the other dimer to form a $\mathcal{P} \mathcal{T}$-symmetric system.

The system is described by a $4 \times 4$ Hamiltonian:

$$
H=\left(\begin{array}{cccc}
-i \gamma & \kappa e^{i \Phi / 2} & 0 & J \\
\kappa e^{-i \Phi / 2} & +i \gamma & J & 0 \\
0 & J & 0 & \kappa e^{i \Phi / 2} \\
J & 0 & \kappa e^{-i \Phi / 2} & 0
\end{array}\right)
$$

The eigenenergy of Hamiltonian $H$ is $E_{ \pm, \pm}= \pm \sqrt{\Delta_{1} \pm \sqrt{\Delta_{2}}}$ with $\Delta_{1}=\kappa^{2}+J^{2}-\gamma^{2} / 2$ and $\Delta_{2}=4 J^{2} \kappa^{2} \cos ^{2}(\Phi / 2)-$ $J^{2} \gamma^{2}+\gamma^{4} / 4$.

\section{III. $\mathcal{P} \mathcal{T}$-SYMMETRIC PHASE}

For $\Delta_{2}>0, \Delta_{1}>\sqrt{\Delta_{2}}$, the four energy levels are all real (phase I). For $\Delta_{2}>0$ and $\Delta_{1}^{2}<\Delta_{2}$, two energy levels $\pm \sqrt{\Delta_{1}+\sqrt{\Delta_{2}}}$ are real, and the other two energy levels $\pm \sqrt{\Delta_{1}-\sqrt{\Delta_{2}}}$ form a conjugate pair (phase II). For $\Delta_{2}<0$ or $\Delta_{1}<0$ and $\Delta_{1}^{2}>\Delta_{2}>0$, four energy levels form two conjugate pairs (phase III). In Fig. 2, the phase diagram is plotted for different magnetic fluxes. The blue and cyan curves constitute a $\lambda$ curve; on the left side of the $\lambda$ curve is the exact $\mathcal{P} \mathcal{T}$-symmetric phase with an entirely real spectrum (phase I); on the right side of the $\lambda$ curve is the broken $\mathcal{P} \mathcal{T}$-symmetric phase, which includes one pair (two pairs) of conjugate energy levels in phase II (III). Phase II is a partially broken $\mathcal{P} \mathcal{T}$ phase; phase III is a completely broken $\mathcal{P} \mathcal{T}$ phase. The solid curves are the boundary between different phases. The cyan and green curves represent $\Delta_{2}=0$, which corresponds to the EP2 with a pair of two-state coalescences; the system has two defective eigenstates in this situation. The blue curves represent $\Delta_{1}^{2}=\Delta_{2}$, which corresponds to the EP2 with a two-state coalescence; the system has one defective eigenstate in this situation. The solid green curves in Fig. 2 are the boundary between region I and III, where $\mathcal{P} \mathcal{T}$-symmetry breaking occurs. The dashed green curves in Fig. 2 are the boundary between energy levels being pure imaginary and being complex numbers. In the presence of magnetic flux, both critical coupling and degree of non-Hermiticity decrease. Thus, the synthetic magnetic flux is favorable for the realization of $\mathcal{P} \mathcal{T}$ phase transition and high-order EPs.

To have real energy, $\Delta_{2} \geqslant 0$ is necessary. At $\Delta_{2}=0$, the system is at an EP2 with a pair of two-state coalescences; this happens at

$$
\gamma_{c, 2, \pm}^{2}=2 J^{2} \pm 2 \sqrt{J^{4}-4 J^{2} \kappa^{2} \cos ^{2}(\Phi / 2)},
$$

where the upper two levels and the lower two levels coalesce. The system has two defective eigenstates and its spectrum is a coalesced conjugate pair. Notably, $\gamma_{\mathrm{c}, 2,-}=\gamma_{\mathrm{c}, 2,+}$ at

$$
J_{\mathrm{c}, 2}^{2}=4 \kappa^{2} \cos ^{2}(\Phi / 2) \text {. }
$$

When $J^{2}<J_{\mathrm{c}, 2}^{2}$, the EP2s with a pair of two-state coalescences disappear, and we have $\Delta_{2}>\left(J^{2}-\gamma^{2} / 2\right)^{2} \geqslant 0$. When $J^{2}>$ 

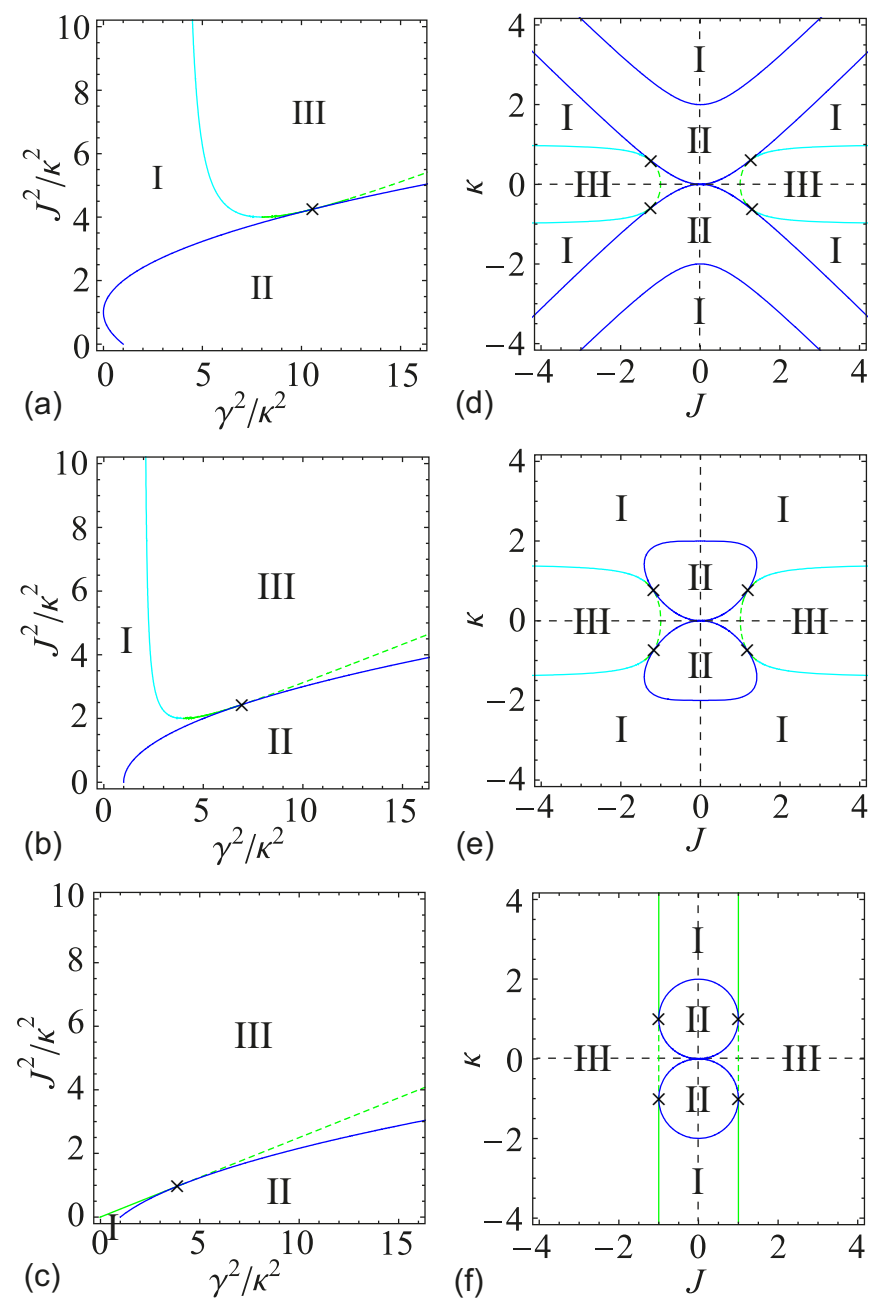

FIG. 2. (a,d) $\Phi=0$, (b,e) $\Phi=\pi / 2$, and (c,f) $\Phi=\pi . \mathcal{P} \mathcal{T}$ phase diagrams are shown in the $\gamma^{2}-J^{2}$ plane for $(\mathrm{a}-\mathrm{c})$. I is the exact $\mathcal{P} \mathcal{T}$ symmetric phase; II and III are the broken $\mathcal{P} \mathcal{T}$-symmetric phases with one and two pairs of conjugate energy levels, respectively. The critical lines of $\gamma_{\mathrm{c}, 1}$ and $\gamma_{\mathrm{c}, 2, \pm}$ are shown in the $\kappa-J$ plane for $(\mathrm{d}-\mathrm{f})$; the couplings are in the unit of a decay rate, $\gamma / 2=1$. The dashed green curve divides the pure imaginary and complex energy levels in region III. The blue solid curves $\left(\gamma_{\mathrm{c}, 1}\right)$ represent the EP2 with one defective eigenstate; the cyan $\left(\gamma_{\mathrm{c}, 2,-}\right)$ and green curves $\left(\gamma_{\mathrm{c}, 2,+}\right)$, both the solid and dashed ones, indicate the EP2 with two defective eigenstates; black crosses mark the high-order EP4s where two types of EP2s coincide.

$J_{\mathrm{c}, 2}^{2}$, then $\Delta_{2}>0$ requires $\gamma^{2}<\gamma_{\mathrm{c}, 2,-}^{2}$ or $\gamma^{2}>\gamma_{\mathrm{c}, 2,+}^{2}$. Notably, $J^{2}>J_{\mathrm{c}, 2}^{2}$ is always satisfied for magnetic flux $\Phi=2 n \pi+\pi$ $(n \in \mathbb{Z})$, where $\gamma_{\mathrm{c}, 2,-}$ vanishes and the system is fragile to nonvanishing gain and loss.

The couplings change the weight of the eigenstate probability distribution. At any weak $J\left(J^{2}<J_{\mathrm{c}, 2}^{2}\right)$, the four resonators are considered as a $\mathcal{P} \mathcal{T}$-symmetric dimer (lower two resonators) weakly coupled to a Hermitian dimer (upper two resonators). The upper and lower levels are always real. Any non-Hermiticity diminishes the mode splitting of the $\mathcal{P} \mathcal{T}$-symmetric dimer; an EP2 $\gamma_{\mathrm{c}, 1}$ with two central energy levels coalesced at energy zero appears at large degree of nonHermiticity. At strong $J\left(J^{2}>J_{\mathrm{c}, 2}^{2}\right)$, the system is considered as two asymmetric dimers coupled through weak nonreciprocal coupling $\kappa e^{ \pm i \Phi / 2}$; the left and right dimers are both nonHermitian, described by an identical $\mathcal{P} \mathcal{T}$-symmetric dimer after removing the overall decay factors $+i \gamma / 2$ and $-i \gamma / 2$.

When $\Delta_{1}^{2}=\Delta_{2} \neq 0$, the central two energy levels coalesce at $E=0$ and the system is at an EP2 of a two-state coalescence with one defective eigenstate. The critical gain and loss must satisfy

$$
\gamma_{\mathrm{c}, 1}^{2}=\left(J^{2}-\kappa^{2}\right)^{2} / \kappa^{2}+4 J^{2} \sin ^{2}(\Phi / 2) .
$$

At $\Phi=2 n \pi \quad(n \in \mathbb{Z})$, the eigenstate for $E=0$ is $\psi_{\sigma}=(-i \sigma \kappa / J,-\kappa / J, i \sigma, 1)^{T}$ with $\sigma=\left|J^{2}-\kappa^{2}\right| /\left(J^{2}-\kappa^{2}\right)$. For the two-state coalescence EP2 with one defective eigenstate, encircling the EP2 in the parameter space with one circle induces the eigenstates to switch [44]; a geometric phase of $\pm \pi$ is accumulated when encircling the EP 2 with two circles that have different chiralities [31]. At $\Phi=2 \pi+2 n \pi(n \in \mathbb{Z})$, the eigenstate for $E=0$ is $\psi_{\sigma}=(-i \sigma \kappa / J, \kappa / J,-i \sigma, 1)^{T}$. The chirality of EP2 is left when $\sigma$ is +1 for $J^{2}>\kappa^{2}$, and the chirality of EP2 is right when $\sigma$ is -1 for $J^{2}<\kappa^{2}$. The chirality of the EPs depends on the competition between the two coupling strengths. The magnetic flux does not change the chirality of EP2s.

The gap between the central two levels closes at $\gamma=0$ for $(\kappa-J)^{2}+4 J \kappa \sin ^{2}(\Phi / 2)=0$. That is only true when $J=$ $\kappa$, and $\Phi=2 n \pi(n \in \mathbb{Z})$. For trivial magnetic flux $\Phi=2 n \pi$ $(n \in \mathbb{Z})$, the critical lines of $\gamma_{\mathrm{c}, 1}$ are open curves in the $J-\kappa$ plane [blue curves in Fig. 2(d)], which are divided into the left chiral EP and the right chiral EP. For nontrivial magnetic flux $\Phi \neq 2 n \pi(n \in \mathbb{Z})$, the critical lines of $\gamma_{\mathrm{c}, 1}$ are closed circles in the $J-\kappa$ plane [blue circles in Figs. 2(e) and 2(f)].

There exist two types of $\gamma_{\mathrm{c}, 1}$. In case I, $\Delta_{1}>0, \Delta_{1}-$ $\sqrt{\Delta_{2}}=0$. The EP2 satisfies $\gamma_{\mathrm{c}, 1}^{2}<2 \kappa^{2}+2 J^{2}$, which requires $J^{2}<J_{\mathrm{c}, 1}^{2}$,

$$
J_{\mathrm{c}, 1}^{2}=2 \kappa^{2} \cos ^{2}(\Phi / 2)+\kappa^{2} \sqrt{4 \cos ^{4}(\Phi / 2)+1} .
$$

In case II, $\Delta_{1}<0, \Delta_{1}+\sqrt{\Delta_{2}}=0$. The EP2 satisfies $\gamma_{\mathrm{c}, 1}^{2}>$ $2 \kappa^{2}+2 J^{2}$, which requires $J^{2}>J_{\mathrm{c}, 1}^{2}$. In case I, when the system parameter crosses $\gamma_{\mathrm{c}, 1}$, the system experiences a $\mathcal{P} \mathcal{T}$ phase transition, while in case II both two sides of $\gamma_{c, 1}$ are $\mathcal{P} \mathcal{T}$-symmetry broken, but with one and two pairs of complex energy levels, respectively.

The system has one (two) defective eigenstate (eigenstates) at $\gamma_{\mathrm{c}, 1}\left(\gamma_{\mathrm{c}, 2, \pm}\right)$. The critical $\gamma_{\mathrm{c}, 2,-}\left(\gamma_{\mathrm{c}, 2,+}\right)$ is illustrated in cyan (green) in Figs. 2(d)-2(f). The curves $\gamma_{\mathrm{c}, 2,-}$ and $\gamma_{\mathrm{c}, 2,+}$ coincide at $\gamma_{\mathrm{c}, 2,-}^{2}=\gamma_{\mathrm{c}, 2,+}^{2}=2 J^{2}$ when $J^{2}=J_{\mathrm{c}, 2}^{2}$ [Eq. (3)]; that is, when a pair of two-state coalescences appear, the energy is $E_{ \pm, \pm}= \pm \kappa$. Note that for $\gamma_{\mathrm{c}, 1}^{2} \geqslant \gamma_{\mathrm{c}, 2,+}^{2} \geqslant \gamma_{\mathrm{c}, 2,-}^{2}$, the curves $\gamma_{\mathrm{c}, 2,+}^{2}$ and $\gamma_{\mathrm{c}, 1}^{2}$ coincide at $J^{2}=J_{\mathrm{c}, 1}^{2}$ [Eq. (5)], where four eigenstates coalesce at zero energy; the system is at an EP4 with three defective eigenstates. The EP4 is the triple point of three different phases: I, II, and III. The critical gain and loss is $\gamma_{\mathrm{EP} 4}^{2}=2 \kappa^{2}\left[1+2 \cos ^{2}(\Phi / 2)+\sqrt{4 \cos ^{4}(\Phi / 2)+1}\right]$, which is $\gamma_{\mathrm{EP} 4}^{2}=4 \kappa^{2}$ for $\kappa=J=1$ as depicted in Fig. 2(f) when $\Phi=\pi$. The only eigenstate at the EP4 is $(1,1, i,-i)^{T}$.

In a Hermitian system, perturbation leads to energy splitting of degenerate states; the resulting energy splitting is proportional to the perturbation $\epsilon$ around Hermitian degeneracies or diabolic points. Operating around EPs or the non-Hermitian 

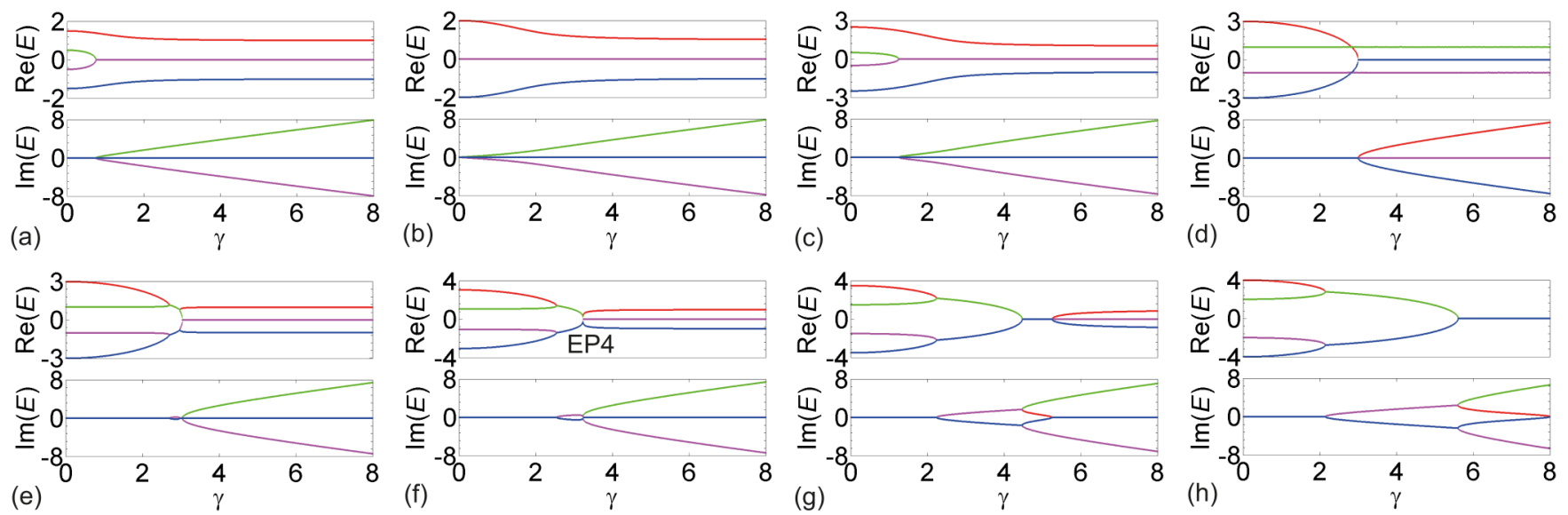

FIG. 3. Energy levels as functions of gain and loss $\gamma$. (a) $J=0.5$, (b) $J=1.0$, (c) $J=1.5$, (d) $J=J_{\mathrm{c}, 2}=2.0$, (e) $J=2.01$, (f) $J=$ $J_{\mathrm{c}, 1}=\sqrt{2+\sqrt{5}} \approx 2.058,(\mathrm{~g}) J=2.5$, and (h) $J=3.0$. Other parameters are $\kappa=1, \Phi=0$.

degeneracies, the energy splitting is more sensitive to the perturbation [27]; in particular, the sensitivity is significantly enhanced for tiny perturbations. The detection sensitivity enhancements at two-order and three-order EPs have been demonstrated in optical systems; the mode frequency splitting induced by the perturbation scales as $\epsilon^{1 / N}$ for an $N$ state coalescence in a non-Hermitian system [28,36]. The sensitivity increases at high-order EPs. In the asymmetric coupled dimers, the EP4 exhibits a mode frequency splitting $E \approx \pm \sqrt{ \pm 2 e^{i 3 \pi / 4}} \epsilon^{1 / 4}$ for perturbation $\epsilon$ on the gain and loss resonators $(i \gamma \rightarrow i \gamma+\epsilon)$. The fourth-root $E \propto \epsilon^{1 / 4}$ results in a significant sensitivity enhancement.

\section{ENERGY-LEVEL STRUCTURE}

The spectrum is symmetric about zero energy; the energy levels exhibit rich structures that are related to the EPs. In Fig. 3, the energy levels are depicted for different values of coupling $J$ at trivial magnetic flux $\Phi=0$ as functions of gain and loss $\gamma$. In the plots from Figs. 3(a)-3(h), the coupling $J$ increases. The coupling $J \leqslant J_{c, 2}=2 \kappa$ is depicted in the upper panel of Figs. 3(a)-3(d), where the system has two real energies. The mode frequency (real part of the energy levels) decreases as gain and loss $\gamma$ increases; the EP $\gamma_{\mathrm{c}, 1}$ appears as the central two levels coalesce at $E_{ \pm,-}=0 . \gamma_{\mathrm{c}, 1}$ in Fig. 3(a) has right chirality. As the coupling increases to $J=\kappa$, the gap between the central two levels closes [Fig. 3(b)]; thereafter, $\gamma_{\mathrm{c}, 1}$ in Fig. 3(c) has left chirality. At $J=J_{\mathrm{c}, 2}=2 \kappa$, two $\gamma$-independent real energy levels $\pm \kappa$ appear [Fig. 3(d)].

For $J^{2}>J_{\mathrm{c}, 2}^{2}$, the critical gain and loss satisfy $\gamma_{\mathrm{c}, 2,-}<$ $\gamma_{\mathrm{c}, 2,+} \leqslant \gamma_{\mathrm{c}, 1}$. The real parts of the uppermost and lowest levels decrease, and the central two levels increase with $\gamma$ until the upper and lower two energy levels coalesce at EP2 $\gamma_{\mathrm{c}, 2,-}$, respectively. When $J_{\mathrm{c}, 2}^{2}<J^{2}<J_{\mathrm{c}, 1}^{2}$, the upper and lower two energy levels coalesce at $\gamma_{\mathrm{c}, 2,-}$ where $\mathcal{P} \mathcal{T}$ transition occurs. The system changes from the $\mathcal{P} \mathcal{T}$-symmetric phase into the broken $\mathcal{P} \mathcal{T}$-symmetric phase; the eigenenergies become two complex conjugate pairs. As $\gamma$ further increases, two pairs of conjugate energy levels bifurcate at $\gamma_{\mathrm{c}, 2,+}$ and the system enters the $\mathcal{P} \mathcal{T}$-symmetric phase once more until the central two levels coalesce at $\gamma_{\mathrm{c}, 1}$ [Fig. 3(e)]; the system reenters the exact
$\mathcal{P} \mathcal{T}$-symmetry region at larger degrees of non-Hermiticity. Therefore, $\gamma<\gamma_{\mathrm{c}, 2,-}$ and $\gamma_{\mathrm{c}, 2,+}<\gamma<\gamma_{\mathrm{c}, 1}$ are in the exact $\mathcal{P} \mathcal{T}$-symmetric phase; $\gamma_{\mathrm{c}, 2,-}<\gamma<\gamma_{\mathrm{c}, 2,+}$ and $\gamma>\gamma_{\mathrm{c}, 1}$ are in the broken $\mathcal{P} \mathcal{T}$-symmetric phase. At $J^{2}=J_{\mathrm{c}, 1}^{2}$, two types of EP2s $\gamma_{\mathrm{c}, 2,+}$ and $\gamma_{\mathrm{c}, 1}$ coincide and all four levels coalesce at $E_{ \pm, \pm}=0$ [Fig. 3(f)].

For $J^{2}>J_{c, 1}^{2}$, the $\mathcal{P} \mathcal{T}$ phase transition occurs only once as $\gamma$ increases. $\gamma<\gamma_{\mathrm{c}, 2,-}$ is the exact $\mathcal{P} \mathcal{T}$-symmetric phase (phase I in Fig. 2); $\gamma_{\mathrm{c}, 2,-}<\gamma<\gamma_{\mathrm{c}, 1}$ is the completely broken $\mathcal{P} \mathcal{T}$-symmetric phase with two conjugate pairs (phase III in Fig. 2): the energy levels are complex when $\gamma_{\mathrm{c}, 2,-}<\gamma<$ $\gamma_{\mathrm{c}, 2,+}$; the energy levels are purely imaginary when $\gamma_{\mathrm{c}, 2,+}<$ $\gamma<\gamma_{\mathrm{c}, 1} ; \gamma>\gamma_{\mathrm{c}, 1}$ is the partially broken $\mathcal{P} \mathcal{T}$-symmetric phase with one conjugate pair (phase II in Fig. 2). The energy levels at large degrees of non-Hermiticity $\gamma \gg J, \kappa$ are in the broken $\mathcal{P} \mathcal{T}$-symmetric region with one pair of real energy levels $E \approx \pm \kappa$ and one pair of conjugate pairs $\pm i \sqrt{\gamma^{2}-\kappa^{2}-2 J^{2}}$ [Figs. 3(g) and 3(h)].

The energy levels for magnetic flux $\Phi=\pi / 2$ are depicted in Fig. 4; the structures for different couplings are similar as illustrated in Figs. 3(c)-3(g). EP2 $\gamma_{\mathrm{c}, 1}$ increases as the coupling
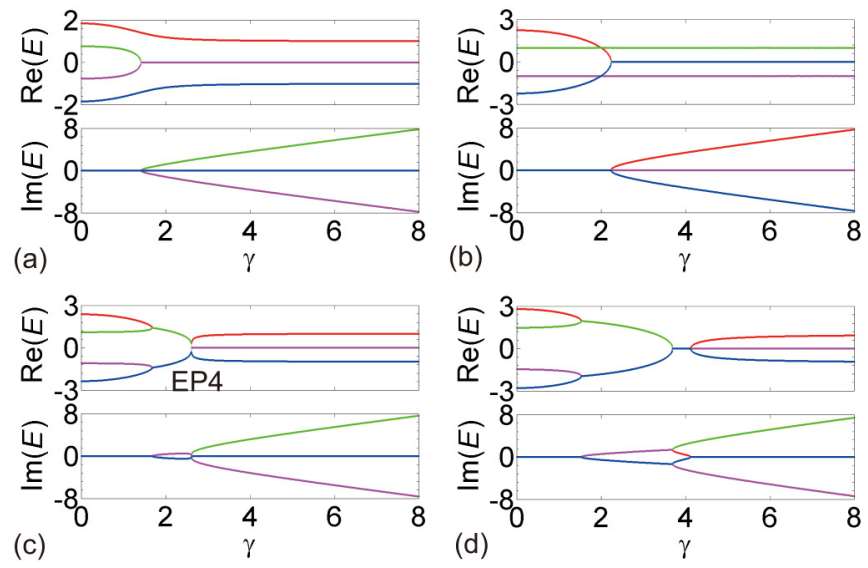

FIG. 4. Energy levels as functions of gain and loss $\gamma$. (a) $J=$ 1, (b) $J=\sqrt{2}$, (c) $J=\sqrt{1+\sqrt{2}} \approx 1.554$, and (d) $J=2$. Other parameters are $\kappa=1, \Phi=\pi / 2$. 
$J$ increases. When $J=\sqrt{2}, \gamma_{\mathrm{c}, 2}$ appears, $\gamma_{\mathrm{c}, 2,-}=\gamma_{\mathrm{c}, 2,+}$. As the coupling $J$ continues to increase, $\gamma_{\mathrm{c}, 2,-}$ and $\gamma_{\mathrm{c}, 2,+}$ split, $\gamma_{\mathrm{c}, 2,-}$ slightly decreases, and $\gamma_{\mathrm{c}, 2,+}$ increases. When $J=$ $J_{c, 1}=\sqrt{1+\sqrt{2}}$, the EP4 occurs and $\gamma_{\mathrm{c}, 2,+}=\gamma_{\mathrm{c}, 1}$. For even larger $J$, the structure of the energy levels remains unchanged. Notably, each EP is a bifurcation point. A typical bifurcation diagram of the spectrum is illustrated in Fig. 4(d). When $\gamma<\gamma_{\mathrm{c}, 2,-}$, the system is in the exact $\mathcal{P} \mathcal{T}$-symmetric phase in phase I; in the region $\gamma_{\mathrm{c}, 2,-}<\gamma<\gamma_{\mathrm{c}, 2,+}$, the system has two conjugation pairs in phase III; in the region $\gamma_{\mathrm{c}, 2,+}<\gamma<\gamma_{\mathrm{c}, 1}$, the energy levels are purely imaginary; in the region $\gamma>\gamma_{\mathrm{c}, 1}$, the system has one pair of real energy and one pair of purely imaginary energy levels in phase II.

\section{TOPOLOGY OF EXCEPTIONAL POINTS}

The phase rigidity for each eigenstate $\psi$ is a useful measure of the mixing of different states [16], which is defined by

$$
r=\left|\left\langle\psi^{*} \mid \psi\right\rangle\langle\psi \mid \psi\rangle\right| \text {. }
$$

In Fig. 5, we depict the phase rigidity and the scaling law for the four eigenstates of Hamiltonian $H$ [Eq. (1)] near the EPs.

In a Hermitian system, the phase rigidity is unity for the real value eigenstate; in a non-Hermitian system, it varies between unity and zero at completely separate resonance and at level coalescence. The situation changes in the presence of magnetic flux; the phase rigidity deviates from unity because the eigenstates are complex even in a Hermitian system. The scaling law of phase rigidity for different EPs is distinct, $r \propto\left|\gamma-\gamma_{\mathrm{c}}\right|^{\nu}$, where the exponent $v$ reflects the topology of those EPs. The phase rigidity may not vanish at EPs in the presence of magnetic flux, where the scaling law transforms to $\left|r-r_{\mathrm{c}}\right| \propto\left|\gamma-\gamma_{\mathrm{c}}\right|^{\nu}$. For trivial magnetic flux $\Phi=2 m \pi$ $(m \in \mathbb{Z})$, the phase rigidity decreases to zero at the EPs.

The magnetic flux and system spectrum are gauge invariant, but the eigenstates depend on the local transformation and the phase rigidities change as local gauge. The scaling exponents are partly independent of the magnetic flux, therefore the phase rigidity can still be used to characterize the topological properties of the EPs. For the symmetrically coupled asymmetric dimers of Eq. (1), the separated $\gamma_{\mathrm{c}, 2,-}, \gamma_{\mathrm{c}, 2,+}$, and $\gamma_{\mathrm{c}, 1}$ are all EP2s. A pair of two-state coalescence points occurs at $\gamma_{\mathrm{c}, 2, \pm}$. The phase rigidity vanishes at $\gamma_{\mathrm{c}, 2,-}$ and $\gamma_{\mathrm{c}, 2,+}$; the system has two defective eigenstates. Two-state coalescence occurs at $\gamma_{c, 1}$, where the system has one defective eigenstate. The phase rigidity at $\gamma_{c, 1}$ does not always vanish, rather it varies as the magnetic flux. The scaling exponents of separated $\gamma_{\mathrm{c}, 2,-}, \gamma_{\mathrm{c}, 2,+}$, and $\gamma_{\mathrm{c}, 1}$ are identical; they are all equal to $1 / 2$ because of the square root relation of EP2. The fractional exponents indicate the eigenstates switch and the nontrivial geometric phases are accumulate when dynamically encircling the EPs $[31,33,34]$. Encircling each separated $\gamma_{\mathrm{c}, 2,-}, \gamma_{\mathrm{c}, 2,+}$, and $\gamma_{\mathrm{c}, 1}$ with two circles, the accumulated geometric phase is $\pm \pi$. Therefore, the eigenstates return to their initial values after encircling EP2 with four circles. The accumulated geometric phases are independent of the magnetic flux.

The topology of EPs changes when they coincide. When the coupling is $J_{\mathrm{c}, 2}, \gamma_{\mathrm{c}, 2,-}$ and $\gamma_{\mathrm{c}, 2,+}$ coincide $\left(\gamma_{\mathrm{c}, 2,-}=\gamma_{\mathrm{c}, 2,+}\right)$ as shown in Fig. 3(d). The accumulated geometric phases
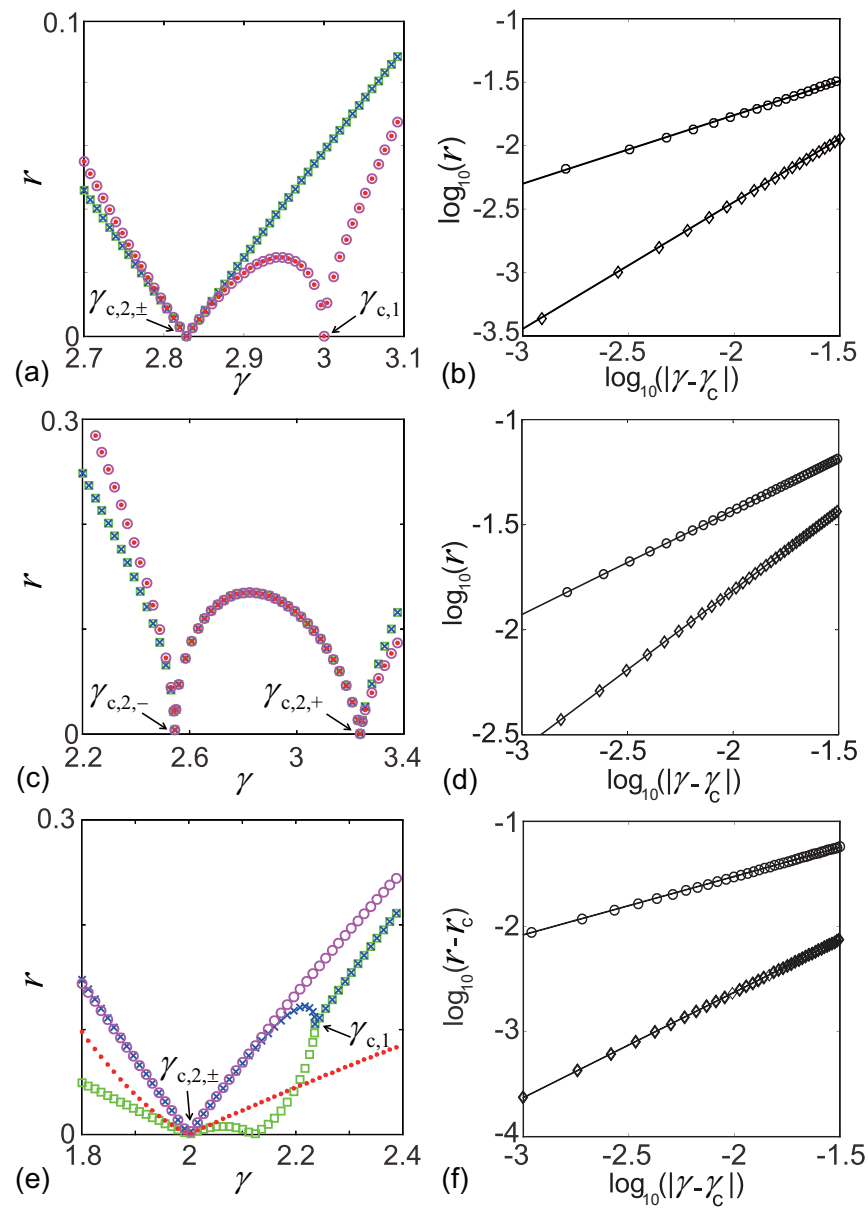

FIG. 5. (a,c,e) Phase rigidity and (b,d,f) the scaling law when EPs coincide for $\kappa=1$. (a,b) $J=J_{\mathrm{c}, 2}=2, \Phi=0$; (c,d) $J=J_{\mathrm{c}, 1}=$ $\sqrt{2+\sqrt{5}}, \Phi=0$; and (e,f) $J=J_{\mathrm{c}, 2}=\sqrt{2}, \Phi=\pi / 2$; corresponding spectra are in Figs. 3 and 4. The fitting exponent approaches 1.0 (0.5) for diamonds (circles) for $\gamma_{\mathrm{c}, 2, \pm}\left(\gamma_{\mathrm{c}, 1}\right)$ in $(\mathrm{b}, \mathrm{f})$ and approaches $0.75(0.5)$ for the diamonds (circles) for $\gamma_{\mathrm{c}, 2,+}\left(\gamma_{\mathrm{c}, 2,-}\right)$ in (d). Energy levels that coalesced at EPs exhibit identical scaling law in (b,d). (f) The green square energy level in (e); the other levels that coalesced at EPs possess the identical scaling exponent.

are related to the magnetic flux; the summation of geometric phases is invariant, being $\pm 2 \pi$ with a pair of symmetric levels $\left(E_{+, \pm}+E_{-, \pm}=0\right)$ when encircling $\gamma_{\mathrm{c}, 2, \pm}$ for one circle. The scaling exponent at $\gamma_{\mathrm{c}, 2, \pm}$ is 1 . The accumulated geometric phase is $\pm \pi$ when encircling $\gamma_{\mathrm{c}, 1}$ with two circles; the scaling exponent at $\gamma_{\mathrm{c}, 1}$ is $1 / 2$. When the coupling is $J_{\mathrm{c}, 1}, \gamma_{\mathrm{c}, 2,+}$ and $\gamma_{\mathrm{c}, 1}$ coincide $\left(\gamma_{\mathrm{c}, 2,+}=\gamma_{\mathrm{c}, 1}\right)$, as depicted in Fig. 3(f). All four energy levels coalesce at energy zero and a high-order EP4 appears, which has an exponent of 3/4. The exponent indicates that four circles are needed to make the energy levels return to their initial values when circling the EP4 in the parameter space. A geometric phase of $\pm 3 \pi$ is accumulated for the eigenstates after encircling the EP4 with four circles. Therefore, the eigenstates return to their initial values after encircling the EP4 with eight circles. AtEP2 $\gamma_{\mathrm{c}, 2,-}$, a geometric phase of $\pm \pi$ is accumulated when encircling $\gamma_{\mathrm{c}, 2,-}$ with two circles; the scaling exponent is $1 / 2$. In Fig. 5, we depict the phase rigidity and the scaling law near the EPs when they 

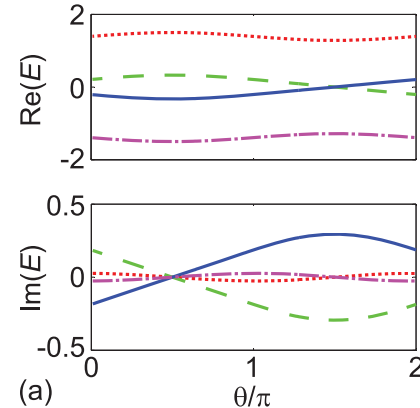

(a)
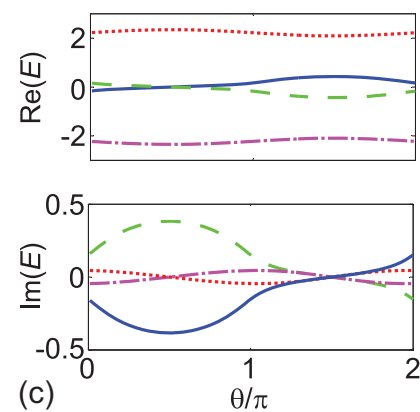

FIG. 6. Encircling $\gamma_{\mathrm{c}, 1}$ at the $\delta-\kappa$ plane, $\delta=\rho \cos \theta$ and $\kappa=1+$ $\rho \sin \theta$. (a,c) Energy levels and (b,d) accumulated geometric phase. The upper panel is for $J=0.5, \gamma=3 / 4$; the lower panel is for $J=$ $1.5, \gamma=5 / 4$. Other parameters are $\Phi=0, \rho=0.1$.

coincide. At coupling $J_{\mathrm{c}, 1}$, the phase rigidities are all zero at EPs and the geometric phases are independent of magnetic flux.

When the coupling $J$ is zero, the system has one critical EP2 at $\gamma=\kappa$. When the coupling $J$ is switched on, the central two levels coalesce at EP2 $\gamma_{\mathrm{c}, 1}$, which decreases as coupling $J$ increases to $J=\kappa$ and increases for $J>\kappa$ (Fig. 3). These are observed in Figs. 3(a)-3(c). $J=\kappa, \gamma=0$ is a diabolic point; the chirality switches when the system parameter crosses this point. The chirality indicates the flow direction; the flow starts from the gain to the loss in clockwise direction or in counterclockwise direction. On the two sides of $J=\kappa$, the coalescence state has two difference chiralities. In Fig. 3(a), the coalescence state is $(1, i,-1 / 2,-i / 2)^{T}$, which has right chirality; in Fig. 3(c), the coalescence state is $(1,-i,-3 / 2,3 i / 2)^{T}$, which has left chirality. The chirality difference of the EPs is reflected from the geometric phase when encircling the EP2 $\gamma_{\mathrm{c}, 1}$, as depicted in Fig. 6.

We consider a detuning $\delta$ on the gain and loss resonators, where the Hamiltonian of Eq. (1) is modified to $H_{1,1}=$ $-(\delta+i \gamma)$ and $H_{2,2}=(\delta+i \gamma)$. The system parameters encircle the EP in the $\delta-\kappa$ parameter plane according to $\delta=\rho \cos \theta$ and $\kappa=1+\rho \sin \theta$, where $\rho$ is the radius and $\theta$ is the argument of the encircling. In the plots, we notice that the central two levels switch after $\theta$ varies $2 \pi$; thus, $\theta$ must be $4 \pi$ to make the energy levels return to their initial values. The Berry connection $i\left\langle\varphi_{j}|\partial| \psi_{j}\right\rangle / \partial k$ is complex, and is defined by the eigenstate $\psi_{j}$ of $H$ with eigenvalue $\varepsilon_{j}$ and corresponding eigenstate $\varphi_{j}$ of $H^{\dagger}$ with eigenvalue $\varepsilon_{j}^{*}[45,46]$. The complex Berry phase is $\left(\oint\left\langle\varphi_{j}\left|\frac{i d}{d k}\right| \psi_{j}\right\rangle d k\right)$ for energy level $j$ after the parameter has slowly swept entire circles in the parameter space. The geometric phases acquired after encircling $\gamma_{\mathrm{c}, 1}$ with two circles in the counterclockwise direction (varying $\theta$ from
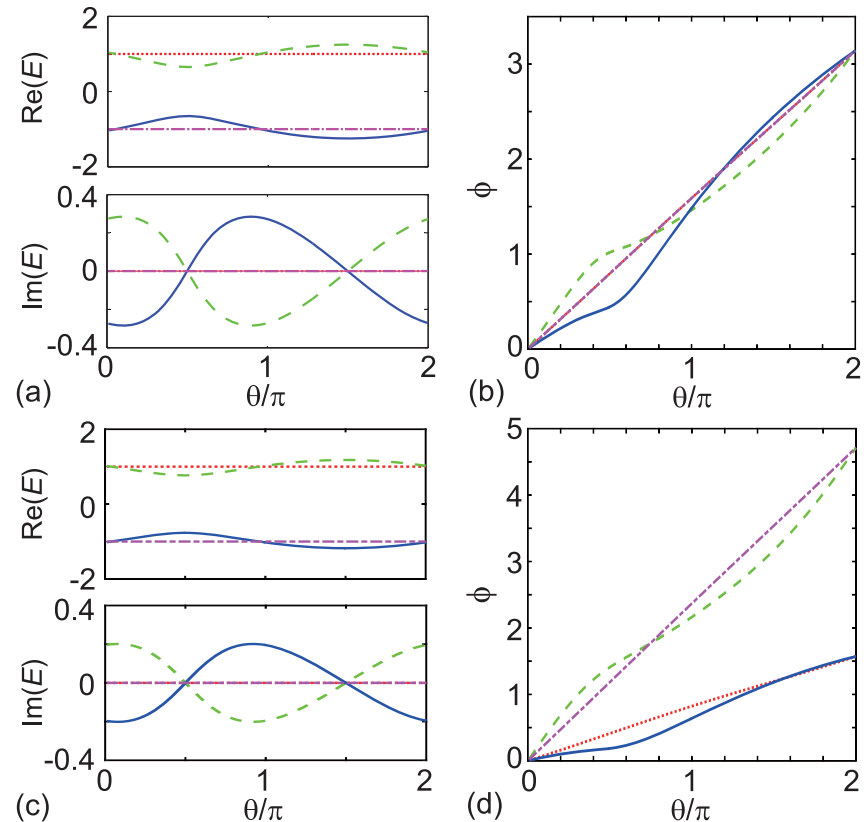

(b)
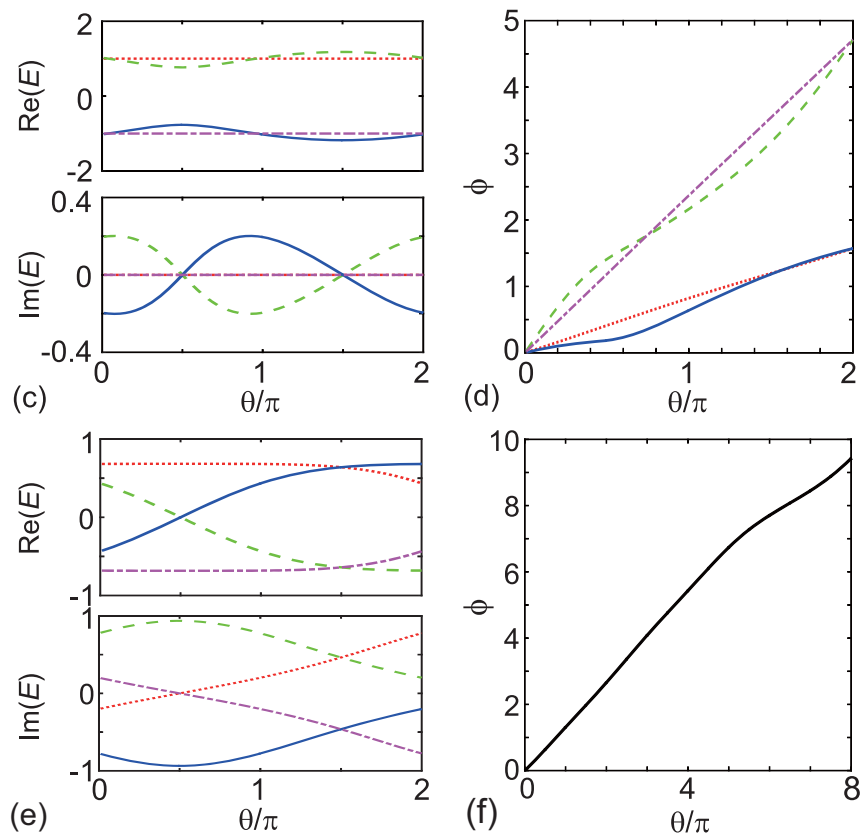

FIG. 7. Encircling $\gamma_{\mathrm{c}, 2,+}$ at the $\delta-\gamma$ plane, $\delta=\rho \cos \theta$ and $\gamma=$ $\gamma_{\mathrm{c}}+\rho \sin \theta$. (a,c,e) Energy levels and (b,d,f) accumulated geometric phase. The upper panel is for $\Phi=0, J=2, \gamma_{\mathrm{c}}=2 \sqrt{2}$; the middle panel is for $\Phi=\pi / 2, J=\sqrt{2}, \gamma_{\mathrm{c}}=2$; the lower panel is for $J=$ $1, \Phi=\pi, \gamma_{\mathrm{c}}=2$. Other parameters are $\kappa=1, \rho=0.1$.

zero to $4 \pi$ ) are $-\pi$ for both two central levels in Fig. 6(b) of the situation shown in Fig. 3(a), where EP2 $\gamma_{\mathrm{c}, 1}$ has right chirality; correspondingly, for the situation in Fig. 3(c), the geometric phases acquired after encircling $\gamma_{c, 1}$ with two circles are $\pi$ as depicted in Fig. 6(d), where EP $2 \gamma_{c, 1}$ has left chirality. Figures 6(b) and 6(d) reflect the chirality difference of $\gamma_{\mathrm{c}, 1}$. For the two central eigenstates, four circles are required to return the eigenstates to their initial values; the top and bottom levels return to themselves when the system parameter encircles $\gamma_{\mathrm{c}, 1}$ with one circle.

In the strong-coupling region $J \geqslant 2 \kappa$, the system experiences more than one EP as $\gamma$ increases from zero. The first EP is an EP2 $\gamma_{\mathrm{c}, 2,-}$ with a pair of two-state coalescences: the upper and lower two levels coalesce. At $J=2 \kappa$, the system does not break $\mathcal{P} \mathcal{T}$ symmetry when the system parameter crosses the first $\operatorname{EP}\left(\gamma_{\mathrm{c}, 2,-}=\gamma_{\mathrm{c}, 2,+}\right)$. The eigenvalues are all real on both sides of $\gamma_{\mathrm{c}, 2, \pm}$. The system at the EP2 $\gamma_{\mathrm{c}, 2, \pm}$ has a pair of two-state coalescences at -1 and +1 , respectively. To reveal the topology of $\gamma_{\mathrm{c}, 2, \pm}$, we consider $\delta=\rho \cos \theta$ and $\gamma=$ $\gamma_{\mathrm{c}, 2, \pm}+\rho \sin \theta$ in the $\delta-\gamma$ parameter plane. Figures 7(a)-7(d) depict the energy levels, the connections, and the accumulated 
geometric phases when encircling $\gamma_{\mathrm{c}, 2, \pm}$ in the $\delta-\gamma$ parameter plane for the situations of $\Phi=0$ and $\pi / 2$, respectively. From the energy levels in Figs. 7(a) and 7(c), we notice that two levels are pinned to \pm 1 , and the other two levels return to themselves after $\theta$ varies $2 \pi$. Therefore, all eigenenergy return to their initial values after the system parameter encircles the EP with one round. The real parts of the Berry phases are depicted in Figs. 7(b) and 7(d). We notice that the accumulated geometric phases are all $\pi$ for $\Phi=0$. The nontrivial magnetic flux in the system changes the Berry connection and the Berry phase. In the $\Phi=\pi / 2$ case, the levels with positive and negative energies accumulate phases of $\pi / 2$ and $3 \pi / 2$; a pair of opposite energy levels accumulate a total phase of $2 \pi$. The EP4 is at $\Phi=\pi, \kappa=J=1$, where all four energy levels coalesce at energy zero. From Fig. 7(e), we notice that all four levels return to their initial values after encircling the EP4 with four complete circles; the geometric phase acquired is $3 \pi$ as shown in Fig. 7(f). Thus, to make the eigenstate recover its initial values, another four circles must encircle EP4.

\section{CONCLUSION}

We investigate a $\mathcal{P} \mathcal{T}$-symmetric system of symmetrically coupled asymmetric dimers. The nonreciprocal coupling induces effective magnetic flux, which provides an extra degree of freedom, which helps with control of the $\mathcal{P} \mathcal{T}$ phase transition. The effective magnetic flux is favorable for the realization of high-order EPs. The EPs and their topology are far richer than those of a $\mathcal{P} \mathcal{T}$-symmetric dimer. Two types of EP2s exist, which possess one and two defective eigenstates, respectively. When EPs coincide, the topology of EPs changes; this is reflected by the scaling exponents and the geometric phases. Although the magnetic flux changes the eigenstates and the phase rigidity, the phase rigidities are always zero for the EP2s with a pair of two-state coalescences. The scaling exponent is independent of the magnetic flux but the geometric phase varies as magnetic flux when EPs have scaling exponent 1. The EP4 is a triple point of different phases; it appears when EP2s with one or two defective eigenstates coincide. The perturbation around the EP4 can result in a fourth-root mode frequency splitting; the response of mode splitting is highly sensitive to the cavity frequency perturbation.

\section{ACKNOWLEDGMENTS}

We acknowledge the support of National Natural Science Foundation of China (Grant No. 11605094) and the Tianjin Natural Science Foundation (Grant No. 16JCYBJC40800).
[1] C. M. Bender and S. Boettcher, Phys. Rev. Lett. 80, 5243 (1998).

[2] P. Dorey, C. Dunning, and R. Tateo, J. Phys. A 34, 5679 (2001).

[3] C. M. Bender, D. C. Brody, and H. F. Jones, Phys. Rev. Lett. 89, 270401 (2002).

[4] A. Mostafazadeh, J. Math. Phys. 43, 205 (2002).

[5] H. F. Jones, J. Phys. A 38, 1741 (2005).

[6] M. Znojil, Phys. Rev. D 78, 025026 (2008).

[7] L. Jin and Z. Song, Phys. Rev. A 80, 052107 (2009).

[8] L. Jin and Z. Song, Phys. Rev. A 81, 032109 (2010).

[9] D. Witthaut, F. Trimborn, H. Hennig, G. Kordas, T. Geisel, and S. Wimberger, Phys. Rev. A 83, 063608 (2011).

[10] Y. N. Joglekar and J. L. Barnett, Phys. Rev. A 84, 024103 (2011).

[11] A. Ruschhaupt, F. Delgado, and J. G. Muga, J. Phys. A 38, L171 (2005).

[12] R. El-Ganainy, K. G. Makris, D. N. Christodoulides, and Z. H. Musslimani, Opt. Lett. 32, 2632 (2007).

[13] Z. H. Musslimani, K. G. Makris, R. El-Ganainy, and D. N. Christodoulides, Phys. Rev. Lett. 100, 030402 (2008).

[14] S. Klaiman, U. Günther, and N. Moiseyev, Phys. Rev. Lett. 101, 080402 (2008).

[15] I. Rotter, J. Phys. A 42, 153001 (2009).

[16] I. Rotter and J. P. Bird, Rep. Prog. Phys. 78, 114001 (2015).

[17] A. Guo, G. J. Salamo, D. Duchesne, R. Morandotti, M. VolatierRavat, V. Aimez, G. A. Siviloglou, and D. N. Christodoulides, Phys. Rev. Lett. 103, 093902 (2009).

[18] C. E. Rüter, K. G. Makris, R. El-Ganainy, D. N. Christodoulides, M. Segev, and D. Kip, Nat. Phys. 6, 192 (2010).

[19] H. Jing, S. K. Özdemir, X.-Y. Lü, J. Zhang, L. Yang, and F. Nori, Phys. Rev. Lett. 113, 053604 (2014).

[20] B. Peng, S. K. Özdemir, F. Lei, F. Monifi, M. Gianfreda, G. L. Long, S. Fan, F. Nori, C. M. Bender, and L. Yang, Nat. Phys. 10, 394 (2014).
[21] B. Peng, S. K. Özdemir, S. Rotter, H. Yilmaz, M. Liertzer, F. Monifi, C. M. Bender, F. Nori, and L. Yang, Science 346, 328 (2014)

[22] L. Feng, Z. J. Wong, R.-M. Ma, Y. Wang, and X. Zhang, Science 346, 972 (2014).

[23] L. Chang, X. Jiang, S. Hua, C. Yang, J. Wen, L. Jiang, G. Li, G. Wang, and M. Xiao, Nat. Photonics 8, 524 (2014).

[24] L. Feng, Y.-L. Xu, W. S. Fegadolli, M.-H. Lu, J. E. B. Oliveira, V. R. Almeida, Y.-F. Chen, and A. Scherer. Nat. Mater. 12, 108 (2013).

[25] E.-M. Graefe and H. F. Jones, Phys. Rev. A 84, 013818 (2011)

[26] C. H. Liang, D. D. Scott, and Y. N. Joglekar, Phys. Rev. A 89, 030102(R) (2014).

[27] J. Wiersig, Phys. Rev. Lett. 112, 203901 (2014).

[28] W. Chen, S. K. Özdemir, G. Zhao, J. Wiersig, and L. Yang, Nature (London) 548, 192 (2017).

[29] C. Dembowski, B. Dietz, H.-D. Gräf, H. L. Harney, A. Heine, W. D. Heiss, and A. Richter, Phys. Rev. E 69, 056216 (2004).

[30] R. Uzdin, A. Mailybaev, and N. Moiseyev, J. Phys. A 44, 435302 (2011).

[31] W. D. Heiss, J. Phys. A 45, 444016 (2012).

[32] H. Menke, M. Klett, H. Cartarius, J. Main, and G. Wunner, Phys. Rev. A 93, 013401 (2016).

[33] J. Doppler, A. A. Mailybaev, J. Böhm, U. Kuhl, A. Girschik, F. Libisch, T. J. Milburn, P. Rabl, N. Moiseyev, and S. Rotter, Nature (London) 537, 76 (2016).

[34] H. Xu, D. Mason, L. Jiang, and J. G. E. Harris, Nature (London) 537, 80 (2016).

[35] K. Ding, G. Ma, M. Xiao, Z. Q. Zhang, and C. T. Chan, Phys. Rev. X 6, 021007 (2016). 
[36] H. Hodaei, A. U. Hassan, S. Wittek, H. Garcia-Gracia1, R. El-Ganainy, D. N. Christodoulides, and M. Khajavikhan, Nature (London) 548, 187 (2017).

[37] K. Fang, Z. Yu, and S. Fan, Phys. Rev. Lett. 108, 153901 (2012).

[38] K. Fang, Z. Yu, and S. Fan, Nat. Photonics 6, 782 (2012).

[39] L. D. Tzuang, K. Fang, P. Nussenzveig, S. Fan, and M. Lipson, Nat. Photonics 8, 701 (2014).

[40] M. Hafezi and P. Rabl, Opt. Express 20, 7672 (2012).
[41] E. Li, B. J. Eggleton, K. Fang, and S. Fan, Nat. Commun. 5, 3225 (2014).

[42] M. Hafezi, Int. J. Mod. Phys. B 28, 1441002 (2014).

[43] M. Hafezi, Phys. Rev. Lett. 112, 210405 (2014).

[44] T. E. Lee, Phys. Rev. Lett. 116, 133903 (2016).

[45] K. Ding, Z. Q. Zhang, and C. T. Chan, Phys. Rev. B 92, 235310 (2015).

[46] H. Zhao, S. Longhi, and L. Feng, Sci. Rep. 5, 17022 (2015). 